\title{
Research on Application Values and Implementation Strategies of Emotional Factors in Physical Education Teaching of Higher Vocational Colleges
}

\author{
Fenglan Fan, Kejun Sun, Lu Sun
}

Beijing Institute of Economic and Management, Beijing, 100102, China

\begin{abstract}
Keywords: Physical education; Higher vocational colleges; Emotional factors
\end{abstract}
\begin{abstract}
Physical education has a systematic characteristic, and its teaching process will be influenced by different factors. Among them, emotional factors are very important, which can optimize teaching effectiveness, deepen emotional communication and enhance thinking ability. After analyzing of the application values of emotional factors in physical education teaching of higher vocational colleges, the paper gives the application strategies of emotional factors in physical education teaching of higher vocational colleges, including maintaining student self-respect, updating teaching content and creating emotional atmosphere to provide some references for the relevant researchers.
\end{abstract}

\section{Introduction}

The addition of emotional factors in higher vocational physical education can improve the quality of teaching and optimize the teaching effect [1]. At present, the development of physical education in higher vocational colleges is facing great challenges and has entered the bottleneck of development. To break this limitation, we need to add emotional factors in teaching and implement different strategies to improve the teaching level of physical education in higher vocational colleges. The diversity and attractiveness of sports can induce people's interest and hobbies in sports activities, and the complexity and variability of sports environment can stimulate people's resilience and behavior. The development of hobbies, interests, behaviors and abilities arouses people's self-consciousness and produces experience to get satisfaction, pleasure, joy and excitement. The collectivism of sports activities can inspire people's social consciousness, enhance their sense of pride, responsibility, self-esteem and self-confidence, and make the emotion socialized, resulting in the spirit of collectivism, such as cooperation, solidarity and fraternity. The competitive nature of sports can stimulate people's enterprising spirit, inspire their will and deepen their emotion, resulting in the optimism, persistence and struggle of optimism. At the same time, the emotion also has the characteristics that cause the body's physiological changes. This characteristic has a positive significance to guide the sports activities. When emotionally excessively excited, nervous or frightened, it tends to breathe faster and shorter, faster heartbeat, higher blood pressure, and facial expression is bitter, panicky, discolored and dysfunctional, so we should appropriately reduce exercise load and psychological burden. When it is necessary to overcome the difficulties and obstacles, emotion can mobilize the inner potential of the organ to complete a certain action. In a heated competition, emotional stimulation leads to increased endocrine and blood sugar content, so that the body can exert strength and complete actions that cannot be done at ordinary times [2].

\section{Application Values of Emotional Factors in Physical Education Teaching of Higher Vocational Colleges}

\subsection{Optimize teaching effectiveness}

The emotion of the teacher and the student is mutual. Vocational college students have their own cognition of the society, and have their own way of doing things, that is, whether they will take the 
initiative to participate in physical education. Interest and motivation in learning are determined by the degree of love for teachers to a certain extent. The application of affective factors is to allow teachers to communicate with students on an equal footing, to weaken the boundaries of two identities, to communicate with each other in a friend's way, and to establish emotional interaction. In this kind of teaching atmosphere, the students will respect the teachers and cooperate with the teachers to complete the teaching task. The management we usually say refers to the design and maintenance of a good environment that enables people to achieve the established goals in this environment. Sometimes there will be some problem behavior in the classroom teaching. The causes of these problems are different, but have been very easy to spread, cause many similar behavior, improper handling will not only cause the contradiction between teachers and classroom discipline problems, interference of teaching activities, but also affect the physical and mental health of students, and even hurt the self-esteem of students and personality. Classroom discipline enables students' group to have a standard code of conduct, ensures the order of classroom learning, and achieves the purpose of teaching. Good emotional communication can enable students to enhance self-discipline and enhance self-discipline, to ensure the smooth progress of teaching. The teacher should timely care and encourage students to care about a word, a cheer encouragement, even a look forward to and support the eyes, will let the students feel encouraged, caring, loved, and then get the infinite confidence, courage and power to to meet the teachers' expectations and requirements to successfully carry out teaching activities and improve the teaching effect [3].

\subsection{Deepen emotional communication.}

There are different emotional exchanges between people and people, which can be between friends and between teachers and students. In the classroom, teachers should give students enough care, do the correct action demonstration for students, guide students carefully, let students learn knowledge, feel the love given by teachers, let the two sides' feelings establish resonance, and narrow the relationship between them. Higher vocational colleges are more than three years. After the end of the three-year system, the establishment of the friendship between the students and the teachers will be the motive force of the students' foreign development. The ways and means of physical education teachers' handling of various classroom behaviors reflect a teacher's educational tact and professional moral cultivation. Effective teaching management desalination of the education marks, edify the temperament of the students, so that students are unwittingly educated. At the same time, physical education teachers should have empathy. This is a way to understand students' feelings and attitudes and observe and think problems from a student's perspective. This ability creates a sense of trust between teachers and students. Teachers should fully understand the problems faced by students, timely and reasonable adjustment and control of their emotions, and look at issues from the position of students, understand their needs and feelings, the students as their friends, put yourself in for the sake of the students, with deep and hot emotion between teachers and students and the noble personality charm and infection education of students. Physical education teachers are also ordinary people, in life will also encounter difficulties and setbacks, emotions also have sadness, depression, trouble. Physical education teachers should consciously abide by the professional ethics of teachers, learn to tolerate, and actively adjust their mentality to face the classroom teaching, with happy and optimistic mood to infect students. Physical education teachers carry out teaching activities under positive emotions. Every word and action is emotional, providing students with a relaxed and harmonious learning environment. It will arouse students' psychological resonance, let them feel the positive attitude and positive energy of teachers, and realize the joy of learning.

\subsection{Enhance thinking ability.}

Emotion can stimulate inspiration, association and phenomenon, and improve the creative thinking ability of people. Emotion is varied and changing. It is the product of rational wings. It enables people to transcend the boundaries between rational and logical thinking, and sublime from perceptual to rational, so that the creativity of thinking can be generated. When the physical education teacher's care is conveyed to the student's mind, students will get good emotional 
experience, and gradually learn to care and care for others with emotional attitude and learn how to control their emotions in daily life. With the development of society, many foreign cultures and theories enter the society, which affects the psychology of college students. Situation of poor psychological ability, ignoring physical education learning, for these students, teachers can use emotional guidance, with the feelings of the students on probation, and actively guide students, gradually into the heart, to eliminate the adverse psychology of the students to quickly adapt to the all-round development of society. From the perspective of psychology, we can inspire students' inspiration by using corresponding emotions, so that they can bring Association and imagination into play and improve their creativity and thinking ability. Emotions are diverse and are constantly changing. This change is a rational command, allowing people to leap from a rational perspective, thereby breaking the boundaries of thinking, subliming ideals into sensibility, and creating corresponding values through the creation of thinking. When we convey the love and care for students to the hearts of students, students will get a relatively good emotional experience. In this process, we will experience their emotional attitudes and start to control their emotions in daily life [4].

\section{Application Strategies of Emotional Factors in Physical Education Teaching of Higher Vocational Colleges}

\subsection{Maintain student self-respect}

Higher vocational students have a strong sense of self-respect and are extremely sensitive. If the teacher does not handle the problem properly at this time, it will hurt their self-esteem, cause negative reaction, weaken the teaching effect and affect the emotion of teachers and students. Teachers should pay attention to safeguarding students' self-esteem and advocate a good teaching atmosphere that teachers and students discuss, learn and promote each other, and use inspiring and guiding methods to enable students to self-discover, self-understand and self-motivate to meet the needs of respect. Understanding is a basic social need in interpersonal communication. Teachers should respect and encourage students who dare to question, think independently and have their own opinions, and help them to analyze and deal with problems together. For some of the weaker students, teachers should be tailored. They do not have a partiality, fair and reasonable, to set an example, to let the students out of admiration from the heart, from the teacher's noble character, feel the harmony of truth, goodness and beauty. Appreciation education emphasizes the evaluation focus on the recognition of the students, so that students feel the attention, acceptance, support, praise and other teachers in the teaching of student success should be fully affirmed, it will not only make their psychological satisfaction, but also enhance the confidence of further study. The best and most direct way is to teach students to use emotional factors. Every action, each explanation, the teacher should be careful to complete, cause the students to resonate, so that the students are willing to accept. A person's emotions can affect others to make the same or associated emotion. Similarly, in the same way, other people's emotions can infect themselves and create the same emotions.

\subsection{Update teaching content}

The occurrence of emotion is not unprovoked. It is produced, developed and mature in concrete practice. Therefore, we should pay attention to the flexibility of teaching methods, the scientific and vividness of teaching contents, and the diversification of teaching methods, so that students can participate in colorful, vivid and interesting activities. This is an important way for teachers to convey emotions and cultivate students' wonderful and rich emotions. Empty preaching, only temporarily effective. Higher vocational students only experience repeatedly all kinds of wonderful emotions in repeated practice, and gradually acquire knowledge in colorful training, which is conducive to the improvement of teaching efficiency. The sports activities situation is various, arranged a beautiful learning environment is a suitable natural environment, it can make students get the feeling of beauty and emotion in learning happy; establish a good sports collective, is a suitable social situation, it can 
make learning in solidarity positive emotion; design a teaching situation, this situation can be virtual, such as stories, assumptions, and can also be simulated, it can make the students in a certain mental environment, Lenovo, imagination, with selfless emotion to learn. Positive and healthy emotion generally have aesthetic significance, so the cultivation of the aesthetic consciousness and aesthetic ability has become an important content of emotional education, the cultivation of aesthetic consciousness and ability of students, so that they can use the aesthetic vision to discover the knowledge contained in the rich content of beauty, to purify the mind and emotions, stimulate students' interest and passion. For example, the environmental beauty of sports equipment layout, the beauty of team formation and the beauty of gymnastic movements, the language and manners of the teachers and students, the behavior beauty of teaching behavior, the movement coordination of movement coordination, rhythm and rhythmic movement are all easy to stimulate students' emotional experience of beauty.

\subsection{Create emotional atmosphere}

Context is the external condition that arouses students' psychological activities. Appropriate situations can arouse people's appropriate feelings, and human emotions are always generated in certain situations. The various factors in the situation, often have a comprehensive effect on the generation of emotions. In sports activities, especially so. The situation of sports activities is varied. Layout of a beautiful learning environment is a suitable natural environment, it can make students get the feeling of beauty and emotion in learning happy; establish a good sports collective, is a suitable social situation, it can make learning in solidarity, positive emotion. A design of situational teaching, this situation can be virtual, such as stories, assumptions, and can also be simulated, it can make the higher vocational students mental environment, Lenovo, imagination, with selfless emotion to learn. This method has the value in the physical education of the lower grade students. PE teachers should pour their emotions into the teaching process, to create a relaxed and harmonious classroom atmosphere, to mobilize the enthusiasm of students and improve the efficiency of classroom teaching. Emotional expression in physical education cannot be phonetic, but it is an important medium for communication and communication between teachers and students. It is the fundamental part of teaching. The teaching of physical education emphasizes practical operation, requires the students to experience their own experience and their own practice, and the emotional communication between teachers and students is often closer and more direct. This kind of emotion will affect the teaching practice, and it is also directly related to the students' active participation. Physical education teachers should convey positive emotions and arouse students' psychological resonance, to promote emotional communication between teachers and students, enhance mutual trust and activate classroom atmosphere to achieve teaching goals smoothly.

\section{Conclusion}

We should take positive measures in accordance with various problems in physical education, combine with the actual situation of higher vocational students, and timely adjust all kinds of psychological contradictions and psychological obstacles caused by pressure of academic and life. At the same time, we should reform the bad factors in the sports management system as soon as possible, change the educational thought and improve the quality of the teachers. If the teacher makes an example, it can affect and affect the students to improve the level of the management of physical education and ensure the smooth realization of the goal of good education.

\section{References}

[1] Zhao Chengyong. Analysis on Role of Emotional Factors in Physical Education Teaching and Its Application Strategies [J]. Journal of Chengdu Aeronautic Polytechnic, 2013, 29(1): 40-41+54.

[2] Liu Ai, Yu Yongguang, Liu Gang. The Role of Emotional Factors in the Teaching of Physical Education and the Implementation Strategy [J]. Yinshan Academic Journal, 2016, 30(1): 122-125. 
[3] Feng Jianli. Discussion on the effect of affective factors in PE teaching in higher vocational colleges [J]. Heilongjiang Science, 2017, 8(5): 62-63.

[4] Hao Xiujiang. The Role of Emotional Factors in Higher Vocational Physical Education and Its Implementation Strategy [J]. Journal of Qiqihar Junior Teachers’College, 2017(6): 99-100. 